University of Wollongong

Research Online

Faculty of Engineering and Information

Faculty of Engineering and Information

Sciences - Papers: Part A

Sciences

2017

Role activity diagram-based discrete event simulation model for healthcare service delivery processes

Nagesh Shukla

University of Wollongong, nshukla@uow.edu.au

John E. Keast

University of Warwick

Darek Ceglarek

University of Warwick

Follow this and additional works at: https://ro.uow.edu.au/eispapers

Part of the Engineering Commons, and the Science and Technology Studies Commons

Research Online is the open access institutional repository for the University of Wollongong. For further information contact the UOW Library: research-pubs@uow.edu.au 


\title{
Role activity diagram-based discrete event simulation model for healthcare service delivery processes
}

\author{
Abstract \\ In case of healthcare systems, discrete event simulations are useful techniques to identify problematic \\ process issues. However, currently available simulation models often use a simplified flow chart as an \\ input which represents patient flow obtained from on on-site observations and interviews complemented \\ with historic patient data. This is insufficient in modelling important interactions between clinical staff, \\ equipment and patients causing the resultant models to be incomplete and unrealistic. This in turn leads \\ to oversimplified outputs from any simulations. This paper presents a systematic methodology for the \\ development of discrete event simulation model from process mapping model based on the Role Activity \\ Diagram (RAD) notations. RAD allows complex collaborative healthcare service delivery processes to be \\ modelled as roles, interactions, actions, and decision questions. The workflow simulation modelling \\ methodology based on RADs includes: (i) development of RAD model of the service delivery process; (ii) \\ data model for RAD based service delivery process; (iii) developing DES model based on RAD; and, (iv) \\ adding dynamic attributes and validating DES model. The methodology is demonstrated through a case \\ study of magnetic resonance (MR) scanning process of radiology department in a large hospital.

\section{Disciplines} \\ Engineering | Science and Technology Studies

\section{Publication Details} \\ Shukla, N., Keast, J. E. \& Ceglarek, D. (2017). Role activity diagram-based discrete event simulation model \\ for healthcare service delivery processes. International Journal of Systems Science, 4 (1), 68-83.
}




\title{
Role Activity Diagram-based Discrete Event Simulation Model for Healthcare Service Delivery Processes
}

\author{
Nagesh Shukla $^{{ }^{*}}$, John E. Keast ${ }^{2}$, Darek Ceglarek ${ }^{2,3}$ \\ ${ }^{1}$ SMART Infrastructure Facility, Faculty of Engineering and Information Sciences, \\ University of Wollongong, Wollongong, NSW, Australia, 2522 \\ ${ }^{2}$ WMG, University of Warwick, Coventry, UK, CV4 7AL \\ ${ }^{3}$ Department of Industrial \& Systems Engineering, University of Wisconsin, Madison, USA, \\ WI 53706 \\ *Corresponding Author: nshukla@uow.edu.au ; Tel: +61 242392329
}




\begin{abstract}
:
In case of healthcare systems, discrete event simulations are useful techniques to identify problematic process issues. However, currently available simulation models often use a simplified flow chart as an input which represents patient flow obtained from on on-site observations and interviews complemented with historic patient data. This is insufficient in modelling important interactions between clinical staff, equipment and patients causing the resultant models to be incomplete and unrealistic. This in turn leads to oversimplified outputs from any simulations. This paper presents a systematic methodology for the development of discrete event simulation model from process mapping model based on the Role Activity Diagram (RAD) notations. RAD allows complex collaborative healthcare service delivery processes to be modelled as roles, interactions, actions, and decision questions. The workflow simulation modelling methodology based on RADs includes: (i) development of $R A D$ model of the service delivery process; (ii) data model for RAD based service delivery process; (iii) developing DES model based on RAD; and, (iv) adding dynamic attributes and validating DES model. The methodology is demonstrated through a case study of magnetic resonance (MR) scanning process of radiology department in a large hospital.
\end{abstract}

Keywords: Healthcare Operations Management, Process Models, Role Activity Diagrams, Discrete Event Simulations 


\section{Introduction}

Due to the crucial role that diagnostic imaging plays in contemporary medicine, physicians from virtually all disciplines who care for patients use diagnostic imaging services. Available estimates indicate that over 1.5 billion imaging procedures were performed in the US (Medicare, 2003) and over 33 million clinical examinations were performed with diagnostic imaging in the UK (Healthcare Commission, 2007). Hence, the increasing needs for imaging services in radiology departments has created considerable bottlenecks in the service delivery processes and resulted in longer patient waiting times (Hillman \& Neiman, 2003). Consequently, the prohibitive costs of imaging devices severely restrict radiology departments from purchasing additional equipment in an effort to enhance patient throughput and reduce waiting times. Hence, improving the efficiency of service delivery process is emerging to be a viable step for radiology departments and a priority of healthcare organizations worldwide (National Research Council, 2009; National Health Services, 2007; Reiner et al., 2002).

Improving the efficiency of imaging service delivery process entails several challenges including optimum allocation and utilization of resources such as medical staffing, reduced non-productive time periods such as patient waiting time, efficiently identifying and eliminating bottlenecks in the service delivery pathway, and streamlining patient flow across radiology department. The majority of research studies in service delivery process improvements have employed process models such as flowcharts (Crabbe et al., 1994), Integrated Definition (IDEF0) (Staccini, et al., 2006), role activity diagrams (RAD) (Shukla, et al., 2014a; Shukla et al. 2009; Patel, 2000), data flow diagrams and value stream mapping (Rother and Shook 2003). These process models are used to document and support service delivery processes in a consistent and uniform manner. Such models aim to support the analysis of a service delivery processes and help in identifying process related problems that can be resolved to achieve the aims of the overall process. Unfortunately, these static models need to address specific dynamic properties (the behavioural aspects, when things are done) if they are going to be useful in helping improvement experts to predict and understand the implications of change, and therefore assist in the making of better decisions.

Few studies related to process improvement have employed discrete event simulation for analysing the service delivery processes (Ewen and Mönch 2014; Lu et al. 2007; Best et al. 2014). The behaviour of the service delivery processes under varying system parameters and scenarios can be studied using simulation modelling. It enables process improvement experts to analyse various improvement scenarios that do not yet exist. Hence, simulation is a tool by 
which current service delivery processes can be understood and any improvement changes can be proposed and analysed beforehand. Once a simulation model of the existing service delivery process is developed, various "what if” questions can be investigated to predict the performance of process produced by change.

The introduction of process modelling approaches has improved the application of simulation modelling for process improvements. However, process improvement experts perceive simulation modelling as a complex tool and hence rarely use these techniques for process analysis (Harrel, 1996; Hulpic, 1998). About half of the process improvement projects failed to deliver the expected benefits (Hammer and Champy, 1994). This can be explained by the lack of tools to evaluate the (redesigned) process performance before its implementation (Paul et al. 1998). Otherwise, any challenges arising from the process improvement will only appear once the redesigned processes are implemented which is costly to correct. Thus, it is necessary to understand and assess how the process will behave when changes have been made before actually implementing them.

Over the past few years, new tools have been developed for process modelling but mostly without addressing their dynamic aspects (Shukla et al. 2014a; Shukla et al. 2014b; Staccini et al. 2006). Most of the tools only capture the complex description about service delivery processes with graphical representations. The study presented in Shukla et al. (2014b) used process models and staff electronic tracking information to understand and highlight unwarranted variations in healthcare. Many of these tools perform some kind of analysis on the process based on the techniques selected for the process representation. However, few of them have been integrated with any form of simulation software (Kettinger et. al. 1997; Lingineni et al. 1995). As a result, double effort is needed in the modelling process if an integrated analysis of the organizational processes is needed. First, the process has to be modelled using a process modelling tool. Then the analyst or decision maker has to start all over again from scratch and model the process using simulation software even though most of the process definition has already been captured with the process models. Therefore, this paper provides an integrated approach which considers both the static and dynamic aspects to analyse service delivery processes. The main contributions of this paper are: (i) to propose a methodology to integrate process models such as role activity diagrams with the discrete event simulation (DES) models, (ii) to develop a set of software tools to aid integration between the process models and the DES, and, (iii) to implement proposed methodology on the real case study on improving magnetic resonance scanning process in a large UK hospital. 


\section{Literature Review}

Researchers in different domains have used tools from their areas such as business processes, operations research and industrial engineering for improving service delivery processes. Each of them has some advantages in improving the processes under consideration. However, there is lack of interdisciplinary approaches used for analysis (Kettinger et al. 1997; Lingineni et al. 1995).

As the first step, the procedural information about the process (i.e. how things are done in service delivery process) is to be acquired (Shadbolt and Milton, 1999). This procedural information is largely qualitative and is acquired from hospital information systems, patient notes, clinician interviews, time and motion studies, and subject matter experts (Shukla et al. 2014a; Crabbe et al. 1994; Miller et al. 2006; White 2005a, 2005b). This type of procedural information is generally used to develop process maps which can be flowcharts (Crabbe et al. 1994), data flow diagrams (Stevens et al. 1974), role activity diagram (RAD) (Shukla et al. 2014a; Shukla et al. 2009; Patel 2000), Integrated Definition for Function Modelling (IDEF) (Staccini et al. 2006); value stream mapping (Rother and Shook 2003) to examine service delivery process for efficiency improvements. However, the resulting process model from these approaches is a static model which lacks the ability to analyse/evaluate different improvement scenarios before being implemented into the existing process.

Different research studies have used dynamic models based on discrete-event simulation to analyse service delivery processes (Duguay and Chetouane 2007; VanBerkel and Blake 2007). The discrete-event simulation (DES) model allows building dynamic models of the service delivery process. Simulation models include scenario based analysis to identify any bottlenecks or inefficiencies. Majority of these simulation models were developed based on inbuilt flow diagrams readily provided by commercial DES software packages such as ARENA, FlexSim, WITNESS. Although, these simulation models were conveniently built in commercial software packages; however, the flow diagrams used were not suitable to represent complexities of healthcare processes. This is partly due to the fact that flow diagrams are best suited to represent sequential processes or non-interaction production processes. In contrast, the healthcare delivery processes are largely collaborative or interactive in nature (among clinicians) (Shukla et al. 2014a).

The aforementioned review of process mapping and DES modelling approaches suggest against the use of one technique over other for process improvements. In case of process modelling techniques, there is a limited or no ability to analyse and predict the behaviour of processes in case of changes. However, discrete event simulation models using simplified 
flow diagrams often fail to capture the process complexities. Therefore, both of these methods are to be integrated for detailed process analysis. Table 1 classifies process improvement approaches in the areas of process mapping and DES.

\section{$<<$ Table 1 Here $>>$}

The majority of the simulation models rely on simplified flowchart of the patient flow obtained based on on-site observations and interviews complemented with historic patient data (Duguay and Chetouane 2007; Nogueira et al. 2014; VanBerkel et al. 2007). However, the resulting workflow model is insufficient in representing the inherent complexity of the process to be modelled. The important interactions between clinical staffs, equipment and patients are not represented in simplified models which causes the resulting model to be often incomplete.

This means that there are lack of systematic approaches which can allow both static process modelling to represent process complexities and dynamic process performance evaluation/analysis. With this view, we have developed an integrated modelling methodology for process modelling and simulation. We used the role activity diagram (RAD) as a process modelling technique, which can effectively model complex interactions in the service delivery processes. Also, we have developed a discrete-event simulation modelling method to simulate the process represented in RADs. A systematic approach for generation of RAD models from the raw process data is presented in Shukla et al. (2014a). This paper extends this methodology to generate DES model based on RADs in a quick and reliable manner.

\section{RAD-based DES modelling methodology}

RAD is defined as a process modelling method originally developed for effectively modelling collaborative processes (Ould 2005). In this study, we used RAD as a process mapping technique for developing a DES model of the service delivery process in a hospital. The fundamental concept of building RAD models is proposed in Shukla et al. (2014a) and therefore it is not repeated in this paper. Interested readers are referred to Appendix A for graphical notations that are used in RAD construction.

To efficiently construct the DES model from RAD, a methodology for translating the RAD concepts to DES is required. The following subsection describes a formal procedure to extract and translate the knowledge represented in RADs to build DES models.

\subsection{Development of RAD model of the service delivery process}


The RAD model of the service delivery process is developed based on the modelling methodology where RAD models are generated from staff interviews (Shukla et al. 2014a). This methodology analyses the interview transcripts of the staff involved in the service delivery to identify and represent procedural knowledge. It helps to efficiently and effectively develop complex RAD models of the process without loss of information from staff discussions. The main steps of this methodology are illustrated with the help of Fig. 1.

\section{$<<$ Figure 1 here $>>$}

Step 1 focuses on generating the input data format for the methodology. It identifies the key roles involved in the process and generates the text format of their interviews. The text/terms that are relevant for the construction of RAD model are extracted in Step 2. The extracted terms are then matched with each other to create relationship matrices such as action-type, action-role, interaction-role and others. These matrices are then used to create RAD model (see Step 4). More information about each of these steps can be obtained in Shukla et al. (2014a).

The implementation of the proposed methodology starts with the development of the RAD model for the service delivery process. RAD modelling from clinician interviews is based on software tools developed in Shukla et al. (2014a). The software tools takes the Microsoft Word based interview transcripts and helps in generating RAD models of the service delivery process in Microsoft Visio. The main steps in the development of RADs are as follows:

\section{a. Interview transcript generation based on Microsoft Word}

The qualitative interviews of the staffs involved in the service delivery process are recorded on audio tape as our primary protocol generation technique. Then the recorded interviews are transcribed into Microsoft Word documents. 
$<<$ Figure 2 here $>>$

A software toolbar using Microsoft Word is developed to mark (extract) terms or texts from the transcribed interviews which were relevant for RAD construction (see Fig. 2.a). These markings are done to extract the relevant terms from the transcripts.

\section{b. Relationship builder tool}

After marking relevant texts from the Microsoft Word transcripts, the marked text is exported into a matrix based tool which allows relationships between concepts to be defined (see Fig. 2.b). The matrix based tool helps to define relationships between marked texts by entering the attribute values (i.e. 1 or 0 ) of the matrices.

c. Graphical representation of RAD in MS Visio

The marked texts and their matrix based relationships are then exported to Microsoft Visio where a RAD is automatically generated using the RAD shape library in Visio (see Fig. 2.c). The RAD model once created can be refined by validating it with the staff that were interviewed. Subsequently, new information from key clinicians can also be added manually to the developed RAD to improve the service delivery process model

\subsection{Data model for RAD based service delivery process}

The RAD model developed in previous section consists of the graphical representation of the service delivery process. It represents only the structure of the process, however, it is useful to have comprehensive data model associated with the RADs for analysis and improvements. Therefore, this section details the data model associated with the RAD concepts which can be used for DES modelling and analysis.

The data associated with the RAD concepts are detailed as:

1. Roles: The data associated with roles are as follows:

a. Role Name: This defines the names of roles present in RAD model.

b. Description: It associates the description about role present in RAD model.

c. Responsibilities: It describes the responsibilities that the role must fulfil. This is a kind of job description.

d. Experience: It defines the experience associated with a particular role in RAD. Experience will typically be described as a period of time working in a particular role. 
e. Skills: It defines the skills required to perform the assigned service delivery task. Skills may be acquired by attendance on a training course which does not lead to a formal qualification.

f. Qualification: It associates the qualification with the role in RAD. The qualifications are the formal qualifications that the role performer must have to be eligible/legally allowed to perform the role.

g. Resources: These describe the resources that must be available or are used by the role performer for the duration of the role. Activity resources only need to be available or are used for the duration of the activity.

h. Number of role performers: It defines the number of people involved in performing a role.

i. Base location: It defines the initial location of the performers of the roles. This attribute is helpful in building the DES model of the process.

2. Activity: The data attributes associated with the activity is defined as:

a. Activity Name: This defines the name of the activity represented in the RAD model of the service delivery process.

b. Activity Description: This attribute contains the textual description about the activity.

c. Times: This attribute associates different types of time such as planned, actual, optimistic, mean, and pessimistic time. Each of these time elements can be further classified as following time elements:

i. Time: The main time elements in this category are: time type, duration, value adding time, essential non value adding, non value adding time, queuing time, waiting time, indecision time, rework time, value adding ratio.

d. Resources: This attribute associates the resource required to perform the activity and are therefore required until activity duration. The main elements in this attributes are: resource ID, resource name, its description, and type.

e. Location: This defines the area level locations where the activity is performed.

f. Issues: This attribute records the issues which are affect the activity processing.

g. Tasks: This defines the list of tasks required to perform an activity.

h. Goals: This attribute describes the goals which are achieved by performing the particular activity. 
i. Flow objects: It defines the type of flow objects involved in the activity processing. It can be of four types: input, output, input/output, constraint.

3. Interactions, encapsulated process: The data attributes for interactions and encapsulated process is similar to that of activities (shown in item 2 above). That is, encapsulated process can be treated as an activity with data attributes such as activity name, activity description, times, resources, location, issues, tasks, goals, and flow objects. Similarly, interactions are defined as an activity which requires more than one type of resources. Therefore, it has a name, description, times, resources (more than one), location, issues, tasks, goals, and flow objects.

These are the main data attributes which forms the data model for RAD. The data model are then translated or exported to other domains with the help of eXtensible Markup Language (XML). XML is a set of rules for encoding documents electronically (W3C 2006; Cunningham 2005). The main purpose for the development of XML was to represent arbitrary data structures and convenient data transfer. RAD models can be encoded into XML format by defined a data structure of the RAD concepts. The data model associated with the RADs can be exported in a XML format defined by a schema.

The data entry forms are linked with the RAD symbols in Microsoft Visio to input relevant data. These forms include data attributes defined in subsection 3.2. Each RAD symbol has a supporting data entry form which allows inputting process information. This data is stored as text or XML in Visio shape properties.

The data model for this phase has been informed by the information requirements for the analysis that are to be performed. Figure 3 shows the form which is associated with the RAD activity symbol. Similarly, data forms are associated with other RAD symbols as well which is not showed here for brevity.

Figure 3 illustrates the time data form for the activity and the data about cost, resources, issues, tasks, goals can be added to the RAD model later. The data related to other RAD concepts such as roles, interactions can be also added into the model. The RAD model complimented with data model can be stored as an XML. Figure 4 illustrates XML file and a snapshot of RAD model developed in Microsoft Visio. The XML snapshot shows the data attributes (such as actual or planned) for an activity perform consenting. Due to brevity, full XML notations are not shown in Fig. 4. However, XML files can be requested by contacting the corresponding author. The RAD toolbar is developed in Microsoft Visio which has the functionalities to generate XML file for the RAD model. 


\subsection{Developing DES model based on RAD}

In this section, the mapping between RAD concepts and DES concepts has been done. This is necessary for seamless integration without loss of information and misinterpretation of the process modelled as RAD (Section 3.1 and Section 3.2). Understanding DES elements and identifying its relation with the RAD concepts is essential to translate the RAD data into DES models.

$<<$ Figure 3 here $>>$

$<<$ Figure 4 here $>>$

Based on the mapping defined in this section it is possible to build a simulation model. By following the mapping from RAD to DES model, the structure of the RAD is retained making it easier for the process improvement experts or domain experts in hospitals to understand and become familiar with the simulation model and experiment with it to analyse different process scenarios and runs. The XML file format is used for the RAD to DES translation.

The Anylogic DES software (from XJ Technologies) is used to interface with RAD software. The RAD concepts and DES model (in Anylogic) is discussed first for mapping. Main DES concepts are:

1. Source: The source object generates the flow entities such as in-patients, out-patients, and emergency patients for the service delivery processes. It is often a starting point of a process model. This object generates the entities which may be of generic class Entity or of any user-defined subclass. It also has parameters such as entity arrival types, rates, entities per arrivals, entity shape, and maximum number of arrivals.

2. Sink: The sink object disposes of the flow entities. It often represents the end of the process.

3. Delay: It delays entities for a specified period of time. It represents the processing time taken to perform any particular activity. Multiple entities can be delayed simultaneously and independently. The parameters of this object include a triangular distribution of delay times, capacity, and statistics.

4. Network: This object maintains the network topology and manages resources in Network-Based Modelling. There must be one network object for each network (and there can be multiple networks in one model). Typically, they are used when the 
processes being modelled are going on in a certain physical space and include movement of entities and resources. For example, this can be a hospital or a plant logistics processes.

5. NetworkResourcePool: It defines a set of network resource units in Network Based Modelling. The objects NetworkSeize and NetworkRelease are used to access the resource. These resource units cannot exit the network defined. The parameters for this object are resource type, capacity, and speed (if resource is dynamically moving), home location, and shape for resource.

\section{$<<$ Table 2 here $>>$}

6. NetworkSeize: It seizes a given set of network resources in Network Based Modelling. It further sends the seized resources to a specified location and attaches them to the entity (both optionally). This object may be considered as a Queue for the entities waiting for resources. This object has parameters such as list of resources to be sized, queue capacity, maximum queue capacity, and attach seized resources.

7. NetworkRelease: It releases all or some of network resources previously seized by the flowing entity in Network Based Modelling. A list of network resource pools is used to identify the resources to be released. The parameters generally used here are moving resources, and list of resources to be released.

8. NetworkMoveTo: It moves the entity to a new network location. If any resources are attached to the entity, they will also move with it. The main parameter under this object is the final destination of the network.

9. Split: It creates one or several other entities, for each incoming entity. The new entities created are the exact copy of incoming entity.

10. Combine: It waits for the two entities to arrive and produces a new entity. The new entity may be a "completely new", i.e. a newly constructed object whose properties possibly depend on the original entities, or it may be one of the original entities, again, possibly modified. Combine is used to re-join the entity with its copy created with Split object.

11. SelectOutput: It directs or routes the incoming entities to one of the two output ports depending on probabilistic or deterministic condition. The condition may depend on the entity as well as on any external factors. This is used to sort entities according to a certain criteria, to randomly split the entity flow. The parameters of this object are condition, and selection probability. 
The main concepts of RAD model are mapped with the concepts of DES modelling for translating the process mapping to DES simulation model. Table 2 illustrates the mapping between RAD and DES modelling. It should be noted that some of the RAD concepts are not used for translation into DES. These are - trigger, state, loop, replication, start role, and other work. This is due to complex/ambiguous nature of these RAD concepts and therefore it requires expert judgement for their translation into DES. Table 2 defines the Anylogic objects used to build simulation model from RAD.

The mapping defined in Table 2 is used to translate RAD concepts (in XML format) into DES model in Anylogic. Anylogic stores its data in a proprietary XML format for which there is no published schema. It was thus necessary to generate sample files using the Anylogic graphical user interface and then infer the schema from the sample files. This process is made easier with the use of a tool like Altova's XMLSpy, but although this tool infers a schema automatically from a sample, considerable manual intervention was still required. Once the schema for the sending and receiving systems are available, it should be possible using a tool such as Altova Mapforce or Biztalk Mapper to generate a translation style sheet (XSLT) using a graphical drag and drop approach. However, the need to translate the Globally Unique Identifiers (GUIDs) from one format to another, the recursive nature of some elements in the Anylogic file and the use of GUIDs in both schema to provide cross referencing between elements, e.g. the from and to links for connectors reference the start and end activity IDs, make this difficult if not impossible. The approach adopted therefore was to use the schema and a Microsoft visual studio to generate a class library corresponding to the Anylogic Schema with serialisation/deserialisation methods built in. Software has then been written to transfer data from RAD classes to the Anylogic classes. The Anylogic data structure is then serialised to provide an Anylogic input file. Currently, the export is only one way i.e. from RAD to Anylogic. After the DES model in Anylogic is created, the dynamic parameters in Anylogic simulation model is provided in following step.

\subsection{Adding dynamic attributes and validating DES model}

The XML document for DES model created in the subsection 3.3 is analysed to eliminate any errors incompatible with the DES modelling. Largely, these errors are due to incomplete information about the dynamic attributes of the DES objects. The dynamic attributes are probabilities of occurrence of several events, arrival rates, capacities of the queues, simulation duration attribute. The DES model generated is analysed for compatibility issues 
such that a comprehensive DES model of the service delivery process is developed based on RAD.

1. Dynamic presentation information: The main presentation objects required, i.e., representations of the flow entities and role/resource objects are created automatically using default shapes and different colours. However, these aspects of the shapes can be customised. Currently, the location shapes created automatically needs to be moved to the appropriate position on the workplace floor layout. Further, the size of locations on the image and movement paths between the locations needs to be defined manually.

2. Linking presentation template with the DES objects: Any dynamic shapes that have been created manually need to be linked with the translated Anylogic objects.

3. Quantitative data to the DES model: The quantitative data is basically the time statistics for the Delay objects, arrival types and rates for Source, resource capacities for NetworkResourcePool, and queue capacities for NetworkSeize. The optimistic, pessimistic and mean durations from the RAD model are used to automatically provide the triangular distribution of times need by the Delay operator. Arrival rates have to be created manually as this data is currently not part of the RAD model. Resource capacities are set from the quantity field in the RAD model and queue capacities default to large values. These may need to be adjusted manually if there are constraints on queue size. This information helps to build a complete Anylogic simulation model which can analyse various improvement scenarios. The visualisation charts for different key performance indices of the service delivery process can be added to the simulation model to measure the effects of changing scenarios. A default chart for utilisation of resources is created automatically.

Any errors in running the simulation model in Anylogic are then manually edited and removed.

The following section presents the application of the developed methodology for building DES model for MR scanning process in a radiology department. The improvement cases are also discussed in next section based on the DES model developed in Anylogic.

\section{Case Study}

The case study involves the Magnetic Resonance Imaging (MRI) scanning service in the radiology department at a large hospital in UK. The radiology unit has two MR scanners to 
fulfil patient scan requests that are generated from other hospital departments. MRI services are provided to patients with the help of radiographers, radiology assistants, porters, radiologists. Two radiographers and one radiology assistant work with each MR scanners to provide the service. Figure 5 shows the physical layout of the MRI scanning area. It shows the two scanners and associated rooms which is used to provide MRI services.

$<<$ Figure 5 here $>>$

The process starts when a patient scan is requested by a consultant in other hospital departments. The request contains some questions on patient health that are to be answered by the radiologist. The radiologist answers those questions based on the patient MR scan and his previous medical data available in hospital information systems. The process ends when the radiologist sends the report containing the answers to consultant's questions.

MRI scan requests from several hospital departments places enormous demand on imaging services. The receptionist schedules the patients for scan procedures on MR machines, each of which has fixed appointment time slots of 20 minutes per area for patients between 9AM - 5PM (excluding one hour of lunch). The area of the patient indicates a class of scan, i.e., head, heart, abdomen, knee, and others. Due to the large demand for scans from different departments and limited number of available patient appointment slots on the MR scanners, patients are expected to wait. According to DoH (2006), no patient should wait more than 18 weeks from GP appointment until their treatment. Timely provision of imaging services plays an important role in achieving the 18 week target. Therefore, the radiology department has to reform their processes to provide MRI services to patients in timely manner.

Patient scanning on MR scanner varies a lot with the age, area to be scanned, health related conditions of patient, and other factors. A patient scan may require contrast injections or sedation injections or any other setup for performing MR scanning effectively. Paediatric patients or claustrophobic patients may require sedation which involves careful medical monitoring, and the scan, therefore, exceeds the 20 minutes/area time allocated for scan. Similarly, disabled patients or patients requiring the contrast injection can also take a large amount of time. However, the physically fit patient without claustrophobia may take less time than 20 min/area. Further, bed ridden inpatients, requiring MR scan, have to be carefully 
transported from wards and prepared for the scanning. The inpatients on pumps, drips, and others require longer preparation time. Hence, there is lot of variations in scanning different types of patients requiring MR scan.

The proposed RAD based DES modelling methodology is applied to model the MR service delivery process. The software tools developed in Section 3 is used to build the simulation model of the service delivery process. The subsections below detail the application of the proposed methodology to MRI scanning process.

\subsection{Development of RAD model for MR service delivery process}

RAD model for MR scanning process is developed based on the modelling methodology developed in Shukla et al. (2014a). Staff representing different roles such as radiographer, receptionist, radiology nurse, porter, and radiologist were interviewed. The face-to-face 30 minute interviews were conducted by project staff. Each staff member was interviewed to detail their involvement in the MRI process. The interviews were recorded. The audio files were transcribed into Microsoft Word (2003). The transcribed interviews were used to develop the RAD model of the MR scanning process (for more information see Shukla et al. 2014a). Figure 6 illustrates the RAD model of the MR scanning process, i.e., from patients arrival at the MR suite at their appointment times until they exit MR suite after scanning.

$<<$ Figure 6 here $>>$

Once the MR scanning process is represented as a RAD, data related to each of the RAD concepts such as time durations are inputted. Then, XML file is generated from RAD model.

\subsection{Develop DES model for MR scanning process}

The RAD toolbar in Microsoft Visio runs the code needed to do the RAD-DES translation. The resulting DES model is opened in Anylogic software and other inconsistencies are manually checked and rectified. The resulting process model in DES software is illustrated in Fig. 7. The translated model in Fig. 7 is based on network based modelling. It comprises of process flow diagram and network resources diagram. The names of the translated concepts are shortened for clarity and to be valid for modelling.

\subsection{Input dynamic attributes in DES model}

Following information is added into the DES model generated in Section 4.3: 
1. Dynamic presentation template of MR scanning process: The shapes associated with roles such as porters, radiology assistants, radiographers, patients (inpatients, outpatients, and emergency patients) are created automatically, but movement paths need to be defined manually. An image of the workplace is added and location objects are adjusted to sit over the MR scanning layout. The main areas in MR scanning layout are reception area, MR rooms, control rooms, changing areas, and a waiting room.

2. Linking presentation template with translated DES objects: The shapes, paths, and areas are linked with objects translated from RAD model such that when a process is simulated the dynamic roles and entities flow in the presentation template. The flow in the presentation template will reflect how the MR scanning process is executed for patients.

\section{Inputting quantitative data to the DES model representing MR scanning process:}

The additional quantitative data needed by the DES model is added. This is mainly arrival rates for source, and any adjustment of queue capacities. The key performance indicator for the MR scanning process is taken to be the utilization rates of the MR scanners as it affects other performance indicators.

Finally, the DES model of MR scanning process is used for analysing various improvement scenarios and its effect on utilization rates of the MR scanning process. The following text illustrates two improvement scenarios and its impact on MR scanning process performance:

A. Having two tables per MR scanner: Due to the fact that only one MR table is present and dedicated to a scanner, therefore, the MR scanner remains idle when a patient scan ends and next patient is being loaded onto the scanner. The disabled outpatients and inpatients come in wheelchairs or on beds for scanning and they require lifting on to the scanner tables, which become free only when the previous patient's scan is finished. To avoid this idle time, an extra MR table is added and its impact on scanner utilization rates are studied. Doing so can reduce wait time. Two scenarios are generated from this improvement case: (i) One scanner table per scanner, and (ii) Two tables per scanner in MR scanning process. These scenarios are simulated and the results are presented in Fig. 8. It shows that having two tables per scanner will be useful to improve the utilization rates from $45 \%$ to $75 \%$.

$<<$ Figure 7 here $>>$ 
B. Performing patient consenting by referring clinicians: The consenting is done for each patients scheduled for MR scan to check the suitability of the patient for MR scan. Patient consenting involves set of questionnaires about metal implants in the patient body. This is due to the negative impact of MR scanning process on patients having metal implants. Further, the radiology staffs are less informed about the patient medical history than the referring clinician or GPs. Therefore, it may be better to shift the consenting to the GP level when patients are referred for scans. By having referring consultants or GPs fill up the consenting form when an MR scan is requested the MR process can save up to 15 minutes of time for each patient. The radiology department could be notified of the status of the already conducted consenting procedure by the referring consultant's or GP's office prior to the scan appointment. Two scenarios are simulated based on the DES model obtained and its impact on utilization rates are studied: i) MR scanning process with consenting procedure; and ii) MR scanning process with short consenting procedure (max. 2 mins). Figure 8 illustrates the improvement realized in both of the scenarios.

\section{$<<$ Figure 8 here $>>$}

\section{Conclusion}

This paper proposed a methodology and software tool to generate the workflow simulation model of complex service delivery process. The methodology employs the RAD models of service delivery process obtained from staff interviews and translates RAD concepts into DES modelling objects. Thus, eliminating the complexity involved in building quick and reliable workflow simulation model. Software toolbar is developed to translate the RAD concepts from Microsoft Visio into DES model in Anylogic software. The methodology is implemented to develop DES model for MR scanning process for analysis and improvements.

The simulation modelling methodology based on static service delivery model can help the process improvement experts for rapid and extensive analysis of the unwarranted variations on a care pathway. The analysis such as bottlenecks identification, low throughput, long waiting times, and low resource utilizations can be easily conducted and their impact on the overall performance of healthcare services can be evaluated. It also helps to perform detailed 
analysis based on RADs and high level process analysis based on simulation models by generating various 'what-if' improvement scenarios. The integration of accurate process model based on RAD of healthcare services with the simulation modelling helps to reduce overall time involved to develop models for hospital simulations. This integration can help decision makers to analyse various improvement scenarios to improve healthcare services. A real case study of MR scanning process of radiology is included to illustrate the integration of simulation modelling with RAD. Two different process improvement scenarios are discussed based on simulation modelling. These scenarios are: (a) two tables per scanner for simultaneous execution of MR scanning and patient preparation; and, (b) patient consenting by referring clinicians. The future research of this work can be on application of proposed methodology in healthcare and other services industries. More work can be done on the developing the flexible data models to import different type of datasets into the DES model. Also, additional work is needed to create a common standardised XML format (containing data model and RAD notations) that can be used in various commercial simulation packages.

\section{ACKNOWLEDGEMENTS}

Authors wish to acknowledge the financial support received from General ElectricHealthcare and Research Councils UK to carry out the study discussed in this paper.

\section{References:}

Best AM, Dixon CA, Kelton WD, Lindsell CJ, Ward MJ. (2014). Using discrete event computer simulation to improve patient flow in a Ghanaian acute care hospital. American Journal of Emergency Medicine, 32(8), 917-22.

Crabbe JP, Frank CL, Nye WW. (1994). Improving report turnaround time: an integrated method using data from a radiology information system. American Journal for Roentgenology, 163, 1503-1507.

Cunningham, Lawrence A. (2005). Language, Deals and Standards: The Future of XML Contracts. Washington University Law Review. SSRN 900616315

Duguay, C., Chetouane, F. (2007). Modelling and Improving Emergency Department Systems using Discrete Event Simulation. Simulation, 83(4), 311-320.

Ewen, H, Mönch, L. (2014). A simulation-based framework to schedule surgeries in an eye hospital. IIE Transactions on Healthcare Systems Engineering, 4(4), 191/208.

Gladwin, B. and Tumay, K., (1994). Modelling business processes with simulation tools. In Proceedings of the 1994 Winter Simulation Conference, 114-121.

Hammer, M., and Champy J. (1994). Reengineering the corporation - a manifesto for business revolution. Nicholas Brealey Publishing, London. 
Harrel, C. and Field K., (1996). Integrating process mapping and simulation. In Proceedings of the 1996 Winter Simulation Conference, 1292-1296.

Healthcare Commission (2007). An improving picture? Imaging services in acute and specialist trusts. Acute Hospital Portfolio Review, 1- 48.

Hillman BJ, Neiman HL. (2003). Radiology 2012: radiology and radiologists a decade hence-a strategic analysis for radiology from the second annual American College of Radiology forum. Radiology, 227, 9-14.

Hlupic V. (1998). Business Process Re-engineering and Simulation: Bridging the Gap. In Proceedings of the European Simulation Multiconference (ESM'98), Ed. by Zobel R. and Moeller D., SCS, 641-645.

Kettinger, W., Teng J. and Guha S. (1997). Business Process Change: A Study of Methodologies, Techniques and Tools. Management Information Systems Quarterly, 21(1), 55-80.

Lingineni, M., Caraway B., Benjamin P. and Mayer R. (1995). A tutorial on PROSIM: A knowledge-based simulation model design. In Proceedings of the 1995 Winter Simulation Conference, 408-412.

Lu, M., Wah-ho Chan, Jian Ping Zhang, Ming Cao (2007). Generic Process Modelling and Simulation Methodology for Integrating Site Layout and Operations Planning in Construction. Journal of Computing in Civil Engineering, 21(6), 453-462.

Medicare, (2003) http://www.medicare.gov/

Miller, M. J., Ferrin,D. M., Shahi, N., LaVecchia, R. (2008). Using RFID Technologies to Capture Simulation Data in a Hospital Emergency Department. Proceedings of the 40th Conference on Winter Simulation, 1637-1644.

National Research Councils (2009). Computational Technology for Effective Health Care: Immediate Steps and Strategic Directions. Editors: Willam W. Stead and Herbert S. Lin; Committee on Engaging the Computer Science Research Community in Health Care Informatics, 1-120.

NHS (2007) "Radiology success factors” Page: 1-1.

Nogueira Jr, L. C., Pinto, L. R., Silva, P. M. S. (2014). Reducing Emergency Medical Service response time via the reallocation of ambulance bases. Health Care Management Science, DOI: 10.1007/s10729-014-9280-4.

Ould, M.A. (2005). Business Process Management: A Rigorous Approach. Meghan-Kiffer Press.

Patel, N. V. (2000). Healthcare Modelling through Role Activity Diagrams for Process-Based Information Systems Development. Requirement Engineering, 5(2), 83-92.

Paul R., Hlupic V., and Giaglis G. M. (1998). Simulation Modeling of Business Processes. In Proceedings of the UK Academy for Information Systems Conference, 311-320.

Reiner, B., Siegel, E., Carrino, J. A. (2002). Workflow optimization: current trends and future directions. Journal of Digital Imaging, 15(3), 141- 152.

Rother, M., Shook, J. (2003). Learning to See - Value-stream Mapping to Create Value and Eliminate Muda. Lean Enterprise Institute, ISBN: 9780966784305.

Shadbolt, N., Milton, N. (1999). From Knowledge Engineering to Knowledge Management. British Journal of Management, 10, 309-322. 
Shukla, N, Keast, J E, Ceglarek, D (2014a). Improved workflow modelling using role activity diagram-based modelling with application to a radiology service case study. Computer Methods and Programs in Biomedicine, 116(3), 274-298.

Shukla, N, Keast, J E, Ceglarek, D (2014b). Modelling variations in hospital service delivery based on real time locating information. Applied Mathematical Modelling, 38(3), 878-893.

Shukla, N., Keast, J., Ceglarek, D, (2009). Role Activity Diagram-Based Workflow Modelling of Imaging Service Delivery Process in Radiology. INFORMS International Conference on Service Sciences.

Staccini, P., Joubert, M., Quaranta, J.F., and Fieschi, M. (2005). Mapping care processes within a hospital: from theory to a web-based proposal merging enterprise modelling and ISO normative principles. International Journal of Medical Informatics, 74(2-4), 335-344

Stevens, W., Myers, G., Constantine, L. (1974). Structured Design, IBM Systems Journal, 13(2), 115-139.

Tumay, K. (1996). Business Process Simulation. Proceedings of the 1996 Winter Simulation Conference, 93-98.

VanBerkel, P. T., and Blake, J. T. (2007). A comprehensive simulation for wait time reduction and capacity planning applied in general surgery. Health Care Management Science, 10, 373-385.

W3C (2006). Extensible Markup Language (XML) 1.1 (Second Edition). Eds. Tim Bray, Jean Paoli, C M Sperberg-McQueen, Eve Maler, Francois Yergeau, John Cowan. Accessed (16 June 2015) http://www.w3.org/TR/xml11/

White K.P., Jr. (2005a). A Survey of Data Resources for Simulating Patient Flows in Healthcare Delivery Systems. In Proceedings of the 2005 Winter Simulation Conference, $926-935$.

White, K.P., Jr. (2005b). Area-Wide Ambulance Diversions: Data Resources for Simulation Modeling. In Proceedings of the 2005 Winter Simulation Conference, Ed. M.E Kuhl, N.M. Steiger, F.B. Armstrong, and J.A. Joines, 926-935. Piscataway, New Jersey.

XJ Technologies http://www.xjtek.com/ 


\section{Appendix A}

Table A.1: Description of RAD concepts and its graphical representation (adapted from Shukla et al. 2014a)

\begin{tabular}{|c|c|c|c|c|}
\hline S. No. & RAD concept & General description & $\begin{array}{l}\text { Examples of } \\
\text { application in } \\
\text { radiology } \\
\text { department }\end{array}$ & $\begin{array}{l}\text { Graphical } \\
\text { notations }\end{array}$ \\
\hline 1. & Role & $\begin{array}{l}\text { Roles have the responsibility for performing a set } \\
\text { of actions and interactions to achieve a goal of } \\
\text { several goals. Roles are not assumed to be the job } \\
\text { titles in the radiology department. Roles can be } \\
\text { performed by organisations, individuals or } \\
\text { machines e.g. computers }\end{array}$ & $\begin{array}{l}\text { Clinical consultants, } \\
\text { porters, nurses, } \\
\text { technicians }\end{array}$ & \\
\hline 2. & Activity & $\begin{array}{l}\text { An activity is a unit of work performed by a } \\
\text { particular role. }\end{array}$ & $\begin{array}{l}\text { Move patient to } \\
\text { changing room, } \\
\text { position patient on } \\
\quad \text { table }\end{array}$ & \\
\hline 3. & Interaction & $\begin{array}{l}\text { An interaction is a synchronous activity where a } \\
\text { number of roles work together to achieve the goals } \\
\text { of the activity. Interactions are taken to include } \\
\text { transfer of something from one role to another. }\end{array}$ & $\begin{array}{l}\text { Pass } X \text {-ray of the } \\
\text { patient from } \\
\text { technician to } \\
\text { radiology consultant }\end{array}$ & \\
\hline 4. & $\begin{array}{l}\text { Part } \\
\text { refinement }\end{array}$ & $\begin{array}{l}\text { The part refinement symbol refers to the work done } \\
\text { simultaneously by a role. This is graphically } \\
\text { represented by single thread of activity dividing } \\
\text { into parallel threads within a role. }\end{array}$ & $\begin{array}{l}\text { Patient booking, and } \\
\text { printing patient non } \\
\text { attendance letters }\end{array}$ & \\
\hline 5. & $\begin{array}{l}\text { Case } \\
\text { refinement }\end{array}$ & $\begin{array}{l}\text { The case refinement is used to represent a decision } \\
\text { question and possible outcomes. }\end{array}$ & $\begin{array}{l}\text { Decision question: } \\
\text { Does patient require } \\
\text { contrast injection? } \\
\text { Outcome: Yes, and no. }\end{array}$ & \\
\hline 6. & Trigger & Trigger is an event that starts the activity thread. & $\begin{array}{l}\text { Arrival of patient scan } \\
\text { request }\end{array}$ & \\
\hline 7. & State & $\begin{array}{l}\text { A state describes what is true either before or after } \\
\text { an action (actions can encompass activities, } \\
\text { interactions, and encapsulated processes). }\end{array}$ & $\begin{array}{l}\text { Scan is finished, } \\
\text { patient can have scan }\end{array}$ & \\
\hline 8. & Loop & $\begin{array}{l}\text { The loop symbol allows a part of a process to be } \\
\text { repeated. The iteration starts at the end of an } \\
\text { activity and goes back to a prior activity. }\end{array}$ & $\begin{array}{l}\text { Multiple attempts to } \\
\text { give an injection }\end{array}$ & \\
\hline 9. & Replication & $\begin{array}{l}\text { This symbol is used to define the nature of the } \\
\text { repetition of certain activities in the process. }\end{array}$ & $\begin{array}{l}\text { Maximum two repeats } \\
\text { in giving injections }\end{array}$ & \\
\hline 10. & $\begin{array}{l}\text { Encapsulated } \\
\text { process }\end{array}$ & $\begin{array}{l}\text { The encapsulated process allows us to represent } \\
\text { complex sub-processes as a separate diagram and } \\
\text { indicating it as a symbol in the main diagram }\end{array}$ & $\begin{array}{l}\text { Perform patient } \\
\text { scanning }\end{array}$ & \\
\hline 11. & Start role & $\begin{array}{l}\text { This symbol is used to initiate a transient role in the } \\
\text { main diagram. }\end{array}$ & $\begin{array}{l}\text { Patient scan request } \\
\text { vetting by radiology } \\
\text { consultant }\end{array}$ & \\
\hline 12. & Other work & $\begin{array}{l}\text { It represents the other work that does not relate to } \\
\text { the main process performed by the role. }\end{array}$ & & \\
\hline 13. & Stop & $\begin{array}{l}\text { This symbol marks the end of one or more threads } \\
\text { to indicate the end point of the process. }\end{array}$ & & \\
\hline
\end{tabular}

